\title{
Thermal effect on vibration of clamped visco-elastic rectangular plate with parabolic thickness variation in both directions
}

\author{
A.K. Gupta ${ }^{\mathrm{a}, *}$, Harvinder Kaur ${ }^{\mathrm{b}}$ and Sanjay Kumar ${ }^{\mathrm{c}}$ \\ ${ }^{a}$ Department of Mathematics, M.S. College, Saharanpur, U.P., India \\ ${ }^{\mathrm{b}}$ Department of Mathematics, Government College, Barwala, Panchkula, Haryana, India \\ ${ }^{\mathrm{c}}$ Department of Mathematics, M.S. College, Saharanpur, U.P., India
}

Received 5 December 2007

Revised 17 August 2008

\begin{abstract}
The analysis presented here is to study the effect of thermal gradient on the vibration of visco-elastic rectangular plate (having clamped boundary condition on all the four edges) of variable thickness whose thickness varies parabolically in both directions. The effect of linear temperature variation has been considered. A frequency equation of plate has been obtained by Rayleigh-Ritz technique with two terms of deflection function. The assumption of small deflection and linear viscoelastic properties of 'Kelvin' type are taken. Deflection and time period corresponding to the first two modes of vibrations for clamped plate have been computed for various combinations of aspect ratio, thermal constants, and taper constants. Numerical computations have been performed for an alloy 'Duralium' and the results obtained are depicted graphically.
\end{abstract}

Keywords: Thermal effect, vibration, visco-elastic, rectangular plate, parabolically thickness, both directions

\section{Introduction}

In the course of time, the study of vibration of plates has acquired great importance in the field of research, engineering and space technology. The visco-elastic behaviors of some materials invigorated scientists for modern designs and analysis techniques and their application to many practical problems. As technology develops new discoveries have intensified the need for solution of various problems of vibrations of plates with elastic or viscoelastic medium.

Since new materials and alloys are in great use in the construction of technically designed structures therefore the application of visco-elasticity is the need of the hour. Tapered plates are generally used to model the structures. Plates with thickness variability are of great importance in a wide variety of engineering applications. Thermal induced vibrations of plates of variable thickness are very useful for research workers in nuclear and chemical engineering. The investigations have resulted in better designing of machine parts and structures subjected to cyclic loading such as gas turbines, jet engine, aircrafts and nuclear power plants.

A study of the literature on vibration problems shows that the visco-elastic plates with thickness variation in two directions has received rather less attention than that of in one direction. Larrondo et al. [10] studied vibrations of simply supported rectangular plates with varying thickness and same aspect ratio cutouts. Free vibrations of rectangular plates of parabolically varying thickness have been investigated by Jain and Soni [9]. Bhatnagar and Gupta [3] discussed thermal effect on vibration of viscoelastic elliptic plate of variable thickness. Laura, Grossi, and

\footnotetext{
*Corresponding author. E-mail: gupta_arunnitin@yahoo.co.in.
} 
Carneiro [11] studied transverse vibrations of rectangular plates with thickness varying in two directions and with edges elastically restrained against rotation.

Singh and Saxena [18] worked on transverse vibration of rectangular plate with bi-directional thickness variation. Free vibration of visco-elastic orthotropic rectangular plates was studied by Sobotka [19]. Sharma [16] worked on some vibration problems of orthotropic plates and shells.

The effect of thermal gradient on the frequencies of an orthotropic plate of linearly varying thickness has been discussed by Tomar and Gupta [22]. Taylor and Govindjee [20] gave solution of clamped rectangular plate problems. Vibration of rectangular plates by the Ritz method was given by Young [23]. Rossi [15] solved the problem of transverse vibrations of thin, orthotropic rectangular plates with rectangular cutouts with fixed boundaries.

Vibration analysis of viscoelastic circular plate subjected to thermal gradient was given by Bhatnagar and Gupta [4]. Cox and Boxer [5] studied the vibration of rectangular plate of uniform thickness with all four-corner point supported. The fundamental frequency of transverse vibration of a clamped rectangular plate of cylindrical orthotropic has been investigated by Bombill and Laura [1].

Filipich et al. [6] considered the rectangular plates with two opposite edges simply supported and the other two with very general boundary conditions and studied the vibrations of such rectangular plates of variable thickness. Tomar and Gupta [21] discussed the effect of thermal gradient on frequencies of an orthotropic rectangular plate whose thickness varies in two directions. Nowacki [14] discussed thermo elasticity in his book. Leissa [12] given the detailed studied on the vibration of plates on his monogram. Nagaya [13] discussed the vibrations and dynamic response of visco-elastic plates on non-periodic elastic supports. Gupta and Khanna [8] solved the problem of vibration of visco-elastic rectangular plate with linearly thickness variations in both directions. Recently, Gupta and Khanna [7] analyzed the vibration of clamped visco-elastic rectangular plate with parabolic thickness variations. Singh and Chakarverty [17] solved the problem for vibration of skew plates using characteristic orthogonal polynomials. Bhat [2] analyses the natural frequencies of rectangular plates using characteristic orthogonal polynomials in Rayliegh- Ritz method.

The aim of present investigation is to study the effect of thermal gradient on the vibration of a clamped visco-elastic rectangular plate whose thickness varies parabolically in both directions, which is an extension of authors' work [7]. Here authors introduced the effect of thermal gradient on their work [7]. It is assumed that the plate is clamped on all the four edges and its temperature varies linearly. The basic differential equation of transverse motion of a visco-elastic rectangular plate of variable thickness is taken from Bhatnagar and Gupta [4]. To determine the frequency equation, Rayleigh-Ritz technique has been used. It is also assumed that deflection is small and linear visco-elastic properties are of 'Kelvin' type, having one spring and one dashpot in parallel.

For various values of thermal gradients, aspect ratio and taper constants, time period and deflection at different points for the first two modes of vibration are evaluated. Results are displayed with graphs. All the material parameters used in numerical calculations have been taken for alloy 'Duralium'.

\section{Equation of motion and constitutive relations}

The equation of transverse motion of a visco-elastic plate of variable thickness, taken in Cartesian co-ordinates, governing density $\rho$, thickness of plate $h$, deflection $w$ and time $t$ is given by Bhatnagar and Gupta [4]:

$$
\frac{\partial^{2} M x}{\partial x^{2}}+2 \frac{\partial^{2} M y x}{\partial x \partial y}+\frac{\partial^{2} M y}{\partial y^{2}}=\rho h \frac{\partial^{2} w}{\partial t^{2}}
$$

The constitutive relation for $M_{x}, M_{y}, M_{y x}$ are given by:

$$
\left.\begin{array}{l}
M_{x}=-\tilde{D} D\left(\frac{\partial^{2} w}{\partial x^{2}}+v \frac{\partial^{2} w}{\partial y^{2}}\right) \\
M_{y}=-\tilde{D} D\left(\frac{\partial^{2} w}{\partial y^{2}}+v \frac{\partial^{2} w}{\partial x^{2}}\right) \\
M_{y x}=-\tilde{D} D(1-v) \frac{\partial^{2} w}{\partial x \partial y}
\end{array}\right\}
$$


where flexural rigidity $D$ is given by:

$$
D=E h^{3} / 12\left(1-\nu^{2}\right),
$$

$\nu$ is the Poisson's ratio, $E$ is Young modulus of elasticity and $\tilde{D}$ is unique Rheological operator .

Substituting these values of $M_{x}, M_{y}$ and $M_{y x}$ from Eq. (2) in Eq. (1):

$$
\begin{aligned}
\tilde{D} & {\left[D\left(\frac{\partial^{4} w}{\partial x^{4}}+2 \frac{\partial^{4} w}{\partial x^{2} \partial y^{2}}+\frac{\partial^{4} w}{\partial y^{4}}\right)+2 \frac{\partial D}{\partial x}\left(\frac{\partial^{3} w}{\partial x^{3}}+\frac{\partial^{3} w}{\partial x \partial y^{2}}\right)+2 \frac{\partial D}{\partial y}\left(\frac{\partial^{3} w}{\partial y^{3}}+\frac{\partial^{3} w}{\partial x^{2} \partial y}\right)\right.} \\
+ & \left.\frac{\partial^{2} D}{\partial x^{2}}\left(\frac{\partial^{2} w}{\partial x^{2}}+v \frac{\partial^{2} w}{\partial y^{2}}\right)+\frac{\partial^{2} D}{\partial y^{2}}\left(\frac{\partial^{2} w}{\partial y^{2}}+v \frac{\partial^{2} w}{\partial x^{2}}\right)+2(1-v) \frac{\partial^{2} D}{\partial x \partial y} \frac{\partial^{2} w}{\partial x \partial y}\right]+\rho h \frac{\partial^{2} w}{\partial t^{2}}=0
\end{aligned}
$$

The solution of Eq. (4) can be given in the form of product of two functions as follows:

$$
w=w(x, y, t)=W(x, y) T(t)
$$

where $W(x, y)$ is the deflection function and $T(t)$ is time function.

Substituting Eq. (5) in Eq. (4) and simplifying, one gets:

$$
\begin{aligned}
& {\left[D\left(\frac{\partial^{4} W}{\partial x^{4}}+2 \frac{\partial^{4} W}{\partial x^{2} \partial y^{2}}+\frac{\partial^{4} W}{\partial y^{4}}\right)+2 \frac{\partial D}{\partial x}\left(\frac{\partial^{3} W}{\partial x^{3}}+\frac{\partial^{3} W}{\partial x \partial y^{2}}\right)+2 \frac{\partial D}{\partial y}\left(\frac{\partial^{3} W}{\partial y^{3}}+\frac{\partial^{3} W}{\partial x^{2} \partial y}\right)\right.} \\
& \left.+\frac{\partial^{2} D}{\partial x^{2}}\left(\frac{\partial^{2} W}{\partial x^{2}}+v \frac{\partial^{2} W}{\partial y^{2}}\right)+\frac{\partial^{2} D}{\partial y^{2}}\left(\frac{\partial^{2} W}{\partial y^{2}}+v \frac{\partial^{2} W}{\partial x^{2}}\right)+2(1-v) \frac{\partial^{2} D}{\partial x \partial y} \frac{\partial^{2} W}{\partial x \partial y}\right] / \rho h W=-\frac{\ddot{T}}{\tilde{D} T}
\end{aligned}
$$

Here superposed dots on $T$ represent the double time derivatives.

Equating both sides of Eq. (6) equal to constant $k^{2}$ we obtain two separate differential equations as follows:

$$
\begin{aligned}
& {\left[D\left(\frac{\partial^{4} W}{\partial x^{4}}+2 \frac{\partial^{4} W}{\partial x^{2} \partial y^{2}}+\frac{\partial^{4} W}{\partial y^{4}}\right)+2 \frac{\partial D}{\partial x}\left(\frac{\partial^{3} W}{\partial x^{3}}+\frac{\partial^{3} W}{\partial x \partial y^{2}}\right)+2 \frac{\partial D}{\partial y}\left(\frac{\partial^{3} W}{\partial y^{3}}+\frac{\partial^{3} W}{\partial x^{2} \partial y}\right)\right.} \\
& \left.+\frac{\partial^{2} D}{\partial x^{2}}\left(\frac{\partial^{2} W}{\partial x^{2}}+v \frac{\partial^{2} W}{\partial y^{2}}\right)+\frac{\partial^{2} D}{\partial y^{2}}\left(\frac{\partial^{2} W}{\partial y^{2}}+v \frac{\partial^{2} W}{\partial x^{2}}\right)+2(1-v) \frac{\partial^{2} D}{\partial x \partial y} \frac{\partial^{2} W}{\partial x \partial y}\right]-\rho h k^{2} W=0
\end{aligned}
$$

and

$$
\ddot{T}+k^{2} \tilde{D} T=0
$$

Equation (7) represents differential equation of transverse motion and Eq. (8) represents differential equation of time function of free vibration of visco-elastic plate of variable thickness.

\section{Formulation of problem and its solution}

Representing $\tau$ and $\tau_{0}$ with the increase in temperatures above the reference temperature at any point at distance $x / a$ and at the end $x=a$ respectively and with the consideration that the temperature of the visco-elastic rectangular plate to vary linearly in one dimension only i.e. along $\mathrm{x}$-axis, $\tau$ may be expressed as:

$$
\tau=\tau_{0}\left(1-\frac{x}{a}\right)
$$

Here, $a$ is the length of the plate.

The temperature dependence of the modulus of elasticity for most of engineering materials, as taken by Nowacki [14], is given by:

$$
E(\tau)=E_{0}(1-\gamma \tau)
$$

where $E_{0}$ is the value of Young's modulus at some reference temperature i.e. $\tau=0$ and $\gamma$ is the slope of variation of $E$ with $\tau$.

Substituting the value of $\tau$ from Eq. (9) into Eq. (10), we have: 


$$
E(\tau)=E_{0}\left[1-\alpha\left(1-\frac{x}{a}\right)\right]
$$

where $\alpha=\gamma \tau_{0}(0 \leqslant \alpha \leqslant 1)$, known as thermal gradient.

The relations for Kinetic energy $P$ and Strain energy $V$ are, as given by Leissa [12], as follows:

$$
P=\left(\frac{1}{2}\right) \rho k^{2} \int_{0}^{a} \int_{0}^{b} h W^{2} d x d y
$$

and

$$
V=\frac{1}{2} \int_{0}^{a} \int_{0}^{b} D\left\{\left(\frac{\partial^{2} W}{\partial x^{2}}\right)^{2}+\left(\frac{\partial^{2} W}{\partial y^{2}}\right)^{2}+2 \nu\left(\frac{\partial^{2} W}{\partial x^{2}} \frac{\partial^{2} W}{\partial y^{2}}\right)+2(1-v)\left(\frac{\partial^{2} W}{\partial x \partial y}\right)^{2}\right\} d x d y
$$

It is also considered that the thickness of the visco-elastic rectangular plate varies parabolically in both directions i.e.

$$
h=h_{0}\left(1+\beta_{1} \frac{x^{2}}{a^{2}}\right)\left(1+\beta_{2} \frac{y^{2}}{b^{2}}\right)
$$

Here $b$ is width of the plate, $\beta_{1}$ and $\beta_{2}$ represent taper constants along $x$-axis and $y$-axis respectively and $h_{0}$ is the thickness of the plate at $x=y=0$.

Making use of Eqs (11) and (14) in Eq. (3), the expression for flexural rigidity $D$ becomes:

$$
D=\frac{1}{12\left(1-\nu^{2}\right)}\left[E_{0} h_{0}^{3}\left\{1-\alpha\left(1-\frac{x}{a}\right)\right\}\left(1+\beta_{1} \frac{x^{2}}{a^{2}}\right)^{3}\left(1+\beta_{2} \frac{y^{2}}{b^{2}}\right)^{3}\right]
$$

Well known Rayleigh-Ritz technique has been utilized here for finding the solution of the present problem with the implication that maximum strain energy must be equal to the maximum kinetic energy, therefore this condition must be satisfied by the present problem:

$$
\delta(V-P)=0
$$

for arbitrary variations of $W$ satisfying relevant geometrical boundary conditions.

\section{Boundary conditions and frequency equation}

The mathematical form of boundary conditions for a rectangular plate having clamped (c) along all the four edges is:

$$
\text { and } \left.\begin{array}{l}
W=\frac{\partial W}{\partial x}=0 \text { at } x=0, a \\
W=\frac{\partial W}{\partial y}=0 \text { at } y=0, b
\end{array}\right\}
$$

and the corresponding two-term deflection function can be written as:

$$
W=\left[\left(\frac{x}{a}\right)\left(\frac{y}{b}\right)\left(1-\frac{x}{a}\right)\left(1-\frac{y}{b}\right)\right]^{2}\left[A_{1}+A_{2}\left(\frac{x}{a}\right)\left(\frac{y}{b}\right)\left(1-\frac{x}{a}\right)\left(1-\frac{y}{b}\right)\right]
$$

which is satisfied by Eq. (17).

Here $A_{1}$ and $A_{2}$ are arbitrary constants, which can be determined by using Eq. (17).

Also consider the non-dimensional variables as:

$$
X=\frac{x}{a}, Y=\frac{y}{a}, \bar{W}=\frac{W}{a}, \bar{h}=\frac{h}{a}
$$

and making use of Eqs (14), (15) and (19) in Eqs (12) and (13) one has: 


$$
P=\left(\frac{1}{2}\right) \rho k^{2} \bar{h}_{0} a^{5} \int_{0}^{1} \int_{0}^{\frac{b}{a}}\left[\left(1+\beta_{1} X^{2}\right)\left(1+\beta_{2} Y^{2} \frac{a^{2}}{b^{2}}\right) \bar{W}^{2}\right] d X d Y
$$

and

$$
\begin{aligned}
V= & C \int_{0}^{1} \int_{0}^{\frac{b}{a}}\{1-\alpha(1-X)\}\left(1+\beta_{1} X^{2}\right)^{3}\left(1+\beta_{2} Y^{2} \frac{a^{2}}{b^{2}}\right)^{3} \\
& \left\{\left(\frac{\partial^{2} \bar{W}}{\partial x^{2}}\right)^{2}+\left(\frac{\partial^{2} \bar{W}}{\partial y^{2}}\right)^{2}+2 \nu\left(\frac{\partial^{2} \bar{W}}{\partial x^{2}} \frac{\partial^{2} \bar{W}}{\partial y^{2}}\right)+2(1-v)\left(\frac{\partial^{2} \bar{W}}{\partial x \partial y}\right)^{2}\right\} d X d Y
\end{aligned}
$$

where $C=\frac{E_{0} \bar{h}_{0}^{3} a^{3}}{24\left(1-v^{2}\right)}$.

Here $X$ varies from 0 to 1 and $Y$ from 0 to $b / a$.

Now substituting the values of $P$ and $V$ from Eqs (20) and (21) in Eq. (16), one gets:

$$
\delta\left(V_{1}-n^{2} k^{2} P_{1}\right)=0
$$

where

$$
\begin{aligned}
V_{1}= & \int_{0}^{1} \int_{0}^{\frac{b}{a}}\left\{(1-\alpha(1-X)\}\left(1+\beta_{1} X^{2}\right)^{3}\left(1+\beta_{2} Y^{2} \frac{a^{2}}{b^{2}}\right)^{3}\right. \\
& \left\{\left(\frac{\partial^{2} \bar{W}}{\partial x^{2}}\right)^{2}+\left(\frac{\partial^{2} \bar{W}}{\partial y^{2}}\right)^{2}+2 \nu\left(\frac{\partial^{2} \bar{W}}{\partial x^{2}} \frac{\partial^{2} \bar{W}}{\partial y^{2}}\right)+2(1-v)\left(\frac{\partial^{2} \bar{W}}{\partial x \partial y}\right)^{2}\right\} d X d Y
\end{aligned}
$$

and

$$
\left.P_{1}=\int_{0}^{1} \int_{0}^{\frac{b}{a}}\left(1+\beta_{1} X^{2}\right)\left(1+\beta_{2} Y^{2} \frac{a^{2}}{b^{2}}\right) \bar{W}^{2}\right] d X d Y
$$

Here

$$
n^{2}=\frac{12 \rho\left(1-v^{2}\right) a^{2}}{E_{0} \bar{h}_{0}^{2}}
$$

is a frequency parameter.

Equation (22) involves two unknown $A_{1}$ and $A_{2}$, arising due to substitution of $W$ from Eq. (18). These unknowns can be evaluated by Eq. (22), as follows:

$$
\left.\begin{array}{c}
\frac{\partial}{\partial A_{1}}\left(V_{1}-n^{2} k^{2} P_{1}\right)=0 \\
\frac{\partial}{\partial A_{2}}\left(V_{1}-n^{2} k^{2} P_{1}\right)=0
\end{array}\right\}
$$

On solving Eq. (25), one has:

$$
\left.\begin{array}{l}
b_{11} A_{1}+b_{12} A_{2}=0 \\
b_{21} A_{1}+b_{22} A_{2}=0
\end{array}\right\}
$$


where $b_{11}, b_{12}, b_{21}, b_{22}$ involve parametric constants and the frequency parameter.

For a non-trivial solution, the determinant of the coefficient of Eq. (26) must vanish. Therefore the frequency equation comes out as:

$$
\left|\begin{array}{l}
b_{11} b_{12} \\
b_{21} b_{22}
\end{array}\right|=0
$$

On solving determinant Eq. (27) one obtains a quadratic equation in $k^{2}$ and through that quadratic equation one gets two values of $k^{2}$. In Eq. (26), on substituting the value of $A_{1}=1$, by choice, one get $A_{2}=-b_{11} / b_{12}$ and hence $W$ becomes:

$$
W=\left[X Y \frac{a}{b}(1-X)\left(1-Y \frac{a}{b}\right)\right]^{2}\left[1+\left(-\frac{b_{11}}{b_{12}}\right) X Y\left(\frac{a}{b}\right)(1-X)\left(1-Y \frac{a}{b}\right)\right]
$$

\section{Time function of vibrations of visco-elastic plate}

The Eq. (8) represents differential equation of time function of free vibration of visco-elastic plate of variable thickness. On solving this differential equation one obtains expression for Time function using unique Rheological operator (which is visco-elastic operator here) $\tilde{D}$. $\tilde{D}$, for Kelvin's Model, is given by:

$$
\tilde{D} \equiv\left\{1+\left(\frac{\eta}{G}\right)\left(\frac{d}{d t}\right)\right\}
$$

where $\eta$ represents visco-elastic constant and $\mathrm{G}$ represents shear modulus. The temperature dependence of these expressions, as used by Nowacki [14], is as follows:

$$
G(\tau)=G_{0}\left(1-\gamma_{1} \tau\right), \eta(\tau)=\eta_{0}\left(1-\gamma_{2} \tau\right)
$$

where $G_{0}$ is shear modulus and $\eta_{0}$ is visco-elastic constant at some reference temperature i.e. at $\tau=0$, slope variation of $\tau$ with $G$ is $\gamma_{1}$ and that with $\eta$ is $\gamma_{2}$.

Making use of Eqs (9) and (19) in Eq. (30),

$$
\text { and } \left.\begin{array}{c}
G=G_{0}\left[1-\alpha_{1}(1-X)\right], \text { where } \alpha_{1}=\gamma_{1} \tau_{0}, 0 \leqslant \alpha_{1} \leqslant 1 \\
\eta=\eta_{0}\left[1-\alpha_{2}(1--X)\right], \text { where } \alpha_{2}=\gamma_{2} \tau_{0}, 0 \leqslant \alpha_{2} \leqslant 1
\end{array}\right\}
$$

Here $\alpha_{1}$ and $\alpha_{2}$ are thermal constants.

On substituting Eq. (31) in Eq. (29), one has:

$$
\tilde{D} \equiv\left\{1+\frac{\eta_{0}\left[1-\alpha_{2}(1-X)\right]}{G_{0}\left[1-\alpha_{1}(1-X)\right]}\left(\frac{d}{d t}\right)\right\}
$$

Therefore

$$
\tilde{D} \equiv\left\{1+p\left(\frac{d}{d t}\right)\right\}
$$

where $p=\frac{\eta}{G}=\frac{\eta_{0}\left[1-\alpha_{2}(1-X)\right]}{G_{0}\left[1-\alpha_{1}(1-X)\right]}$.

Substituting Eq. (33) in Eq. (8), we get:

$$
\ddot{T}+k^{2} p \dot{T}+k^{2} T=0
$$

Equation (34) is a second order differential equation in time function $T$. The solution of which comes out to be

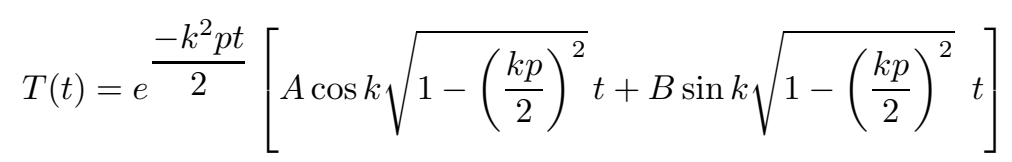

Here $A$ and $B$ are constants of integration.

Equation (35) can be the expressed as: 


$$
T(t)=e^{\theta t}[A \cos \delta t+B \sin \delta t]
$$

where

$$
\theta=-\frac{k^{2} p}{2}
$$

and

$$
\delta=k \sqrt{1-\left(\frac{k p}{2}\right)^{2}}
$$

Assuming initial conditions as:

$$
T=1 \text { and } \dot{T}=0 \text { at } t=0
$$

On using Eq. (37) in Eq. (35), one gets:

$$
A=1 \text { and } B=k^{2} p / 2 \delta=-\theta / \delta
$$

Making use of the values of $A$ and $B$ from Eq. (38) in Eq. (36), one has:

$$
T(t)=e^{\theta t}\left[\cos \delta t+\left(\frac{-\theta}{\delta}\right) \sin \delta t\right]
$$

On substituting Eqs (39) and (28) in Eq. (5), gives deflection w as:

$$
\begin{aligned}
w= & {[X Y(a / b)(1-X)(1-Y a / b)]^{2}\left[1+\left(-b_{11} / b_{12}\right) X Y(a / b)(1-X)(1-Y a / b)\right] } \\
& \times\left[e^{\theta t}\left\{\cos \delta t+\left(\frac{-\theta}{\delta}\right) \sin \delta t\right\}\right]
\end{aligned}
$$

Time period of the vibration of the plate is expressed as:

$$
K=2 \pi / k
$$

where $k$ is given by Eq. (27).

\section{Numerical results and discussions}

For explanation of the problem in detail, computation has been done and the values of time period $K$ and deflection $w$ for a visco-elastic rectangular plate for different values of taper constants $\beta_{1}$ and $\beta_{2}$, thermal gradients $\alpha, \alpha_{1}$ and $\alpha_{2}$ and aspect ratio $\mathrm{a} / \mathrm{b}$ at different points for first two modes of vibrations have been calculated numerically.

In order to discuss the problem for the purpose of numerical calculation, we take the values of relevant physical parameters of 'Duralium', as used by Nagaya [13], which are as follows:

$$
\begin{aligned}
& E=7.08 \times 10^{10} \mathrm{~N} / \mathrm{M}^{2} \\
& G=2.632 \times 10^{10} \mathrm{~N} / \mathrm{M}^{2} \\
& \eta=14.612 \times 10^{5} \mathrm{NS} / \mathrm{M}^{2} \\
& \rho=2.80 \times 10^{3} \mathrm{Kg} / \mathrm{M}^{3} \\
& \nu=0.345 \\
& h_{0}=0.01 \mathrm{M}
\end{aligned}
$$

where $h_{0}$ stands for the thickness of the plate at $x=y=0$.

For accuracy of the results, computations are carried out through $\mathrm{C}^{++}$programming on a PC. These results are represented graphically in Figs 1 to 7 .

Figure 1 depicts the results of time period $\mathrm{K}$ for a preset value of aspect ratio $\mathrm{a} / \mathrm{b}(=1.5)$ and for different thermal gradients $\alpha$ for first two modes of vibration for the subsequent four combinations of $\beta_{1}$ and $\beta_{2}$ :
(i) $\beta_{1}=0.0, \beta_{2}=0.0$;
(ii) $\beta_{1}=0.0, \beta_{2}=0.2$;
(iii) $\beta_{1}=0.4, \beta_{2}=0.0$;
(iv) $\beta_{1}=0.4, \beta_{2}=0.2$ 


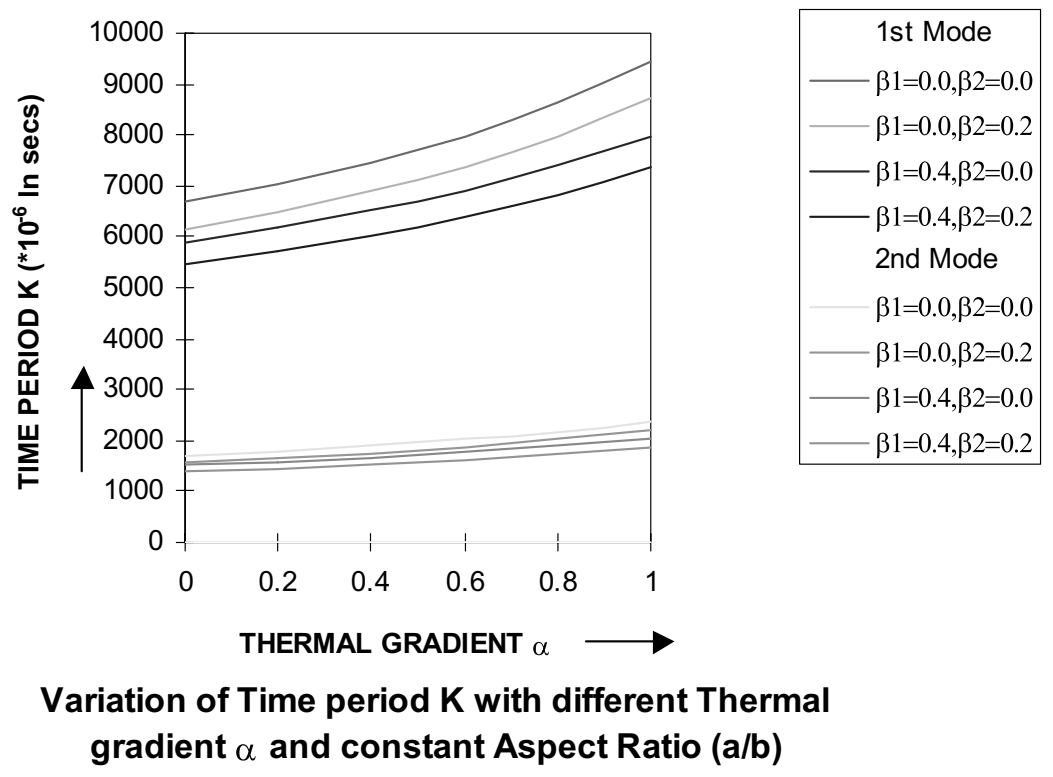

Fig. 1. Variation of Time period K with different Thermal gradient a and constant Aspect Ratio (a/b).

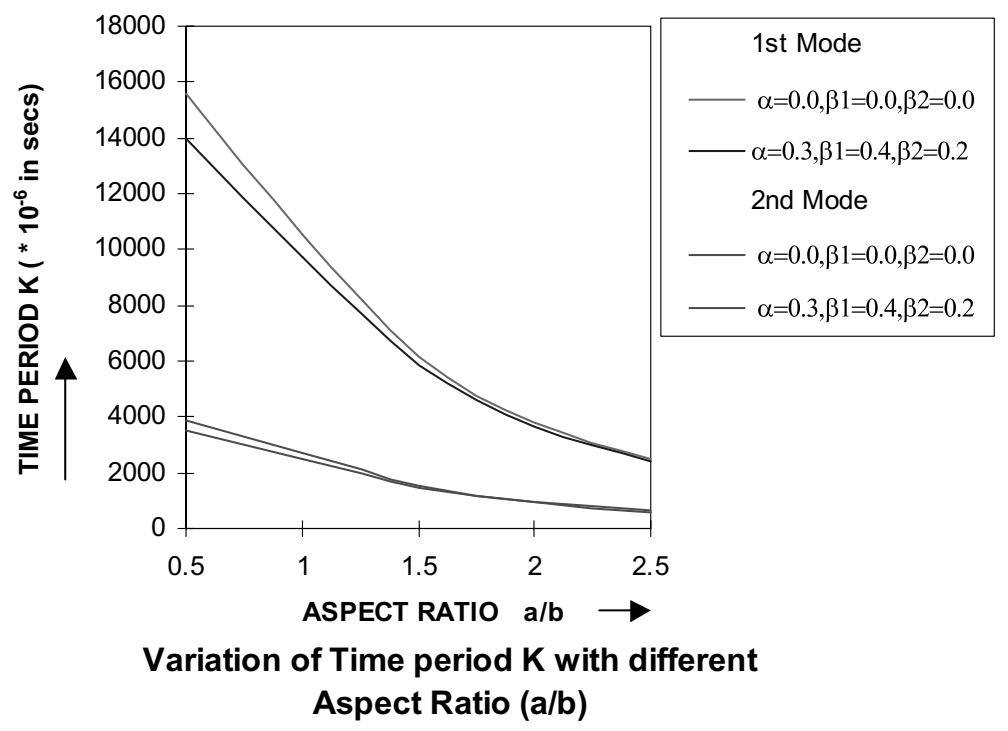

Fig. 2. Variation of Time period K with different Aspect Ratio (a/b).

It can be easily observed from Fig. 1 that as thermal gradient $\alpha$ increases, time period $\mathrm{K}$ increases continuously for both the modes of vibration.

Figure 2 presents the values of time period $\mathrm{K}$ for different values of aspect ratio $\mathrm{a} / \mathrm{b}$ for both the modes of vibration for the following:
(i) $\alpha=0.0, \beta_{1}=\beta_{2}=0.0$
(ii) $\alpha=0.3, \beta_{1}=0.4, \beta_{2}=0.2$

It is clear from Fig. 2 that the value of time period $\mathrm{K}$ decreases as aspect ratio $\mathrm{a} / \mathrm{b}$ increases for both the modes of vibration in both cases.

Figure 3 displays the results of time period $\mathrm{K}$, for first two modes of vibration, for preset aspect ratio $\mathrm{a} / \mathrm{b}$ and for fixed thermal gradient $\alpha(=0.3)$ and for set value of taper constant $\beta_{2}(=0.2)$ for different values of taper constant 


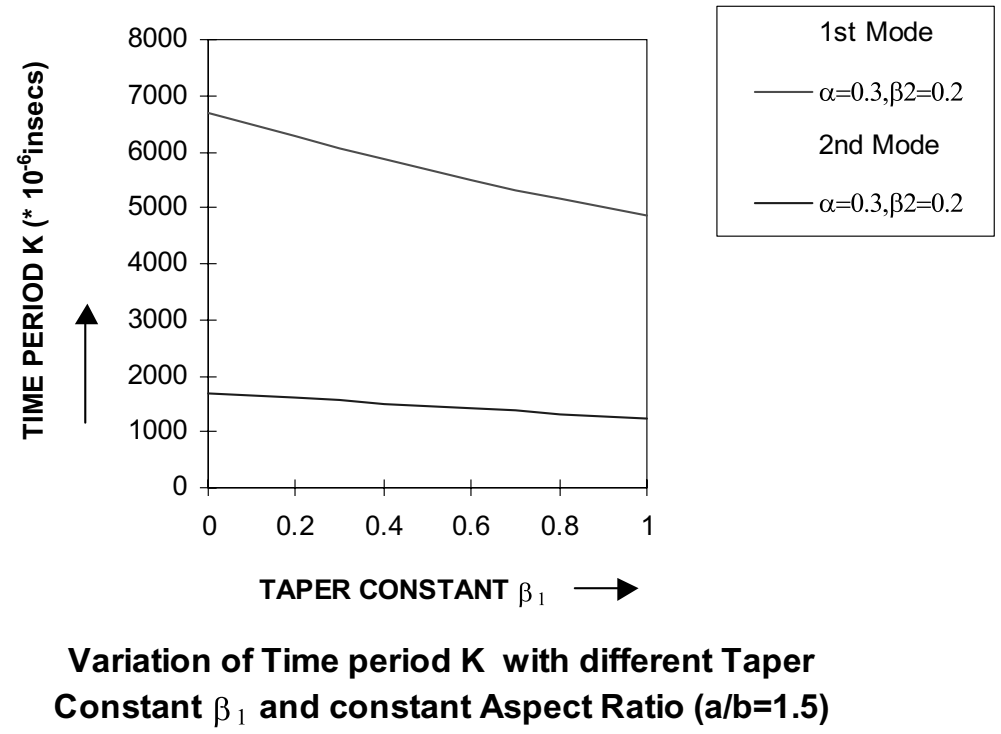

Fig. 3. Variation of Time period $\mathrm{K}$ with different Taper Constant $\mathrm{b} 1$ and constant Aspect Ratio $(\mathrm{a} / \mathrm{b}=1.5)$.
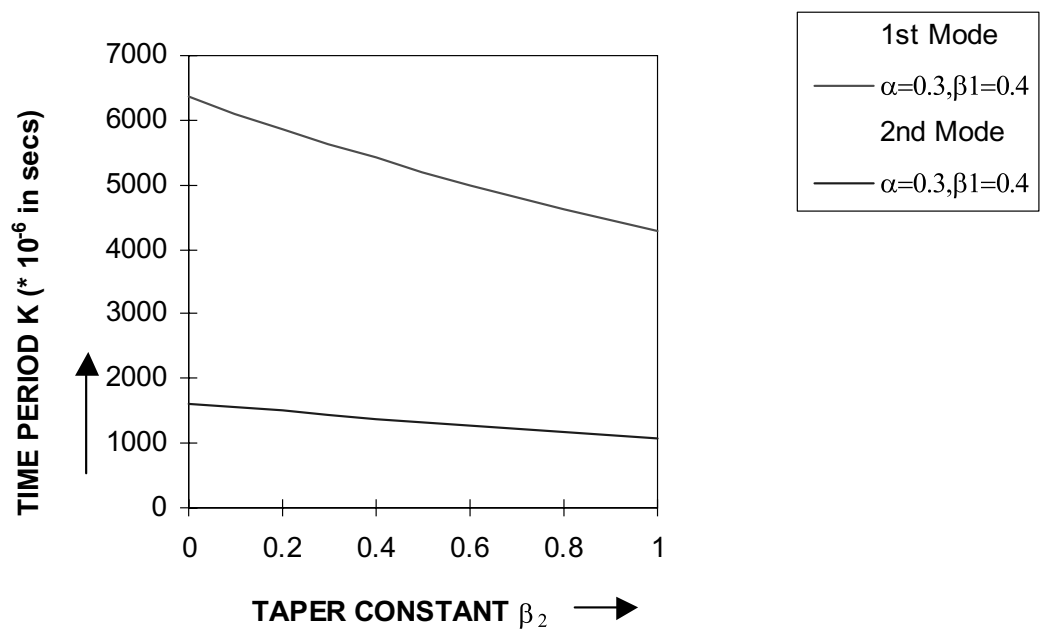

\section{Variation of Time period $\mathrm{K}$ with different Taper Constant $\beta_{2}$ and constant Aspect Ratio (a/b=1.5)}

Fig. 4. Variation of Time period $\mathrm{K}$ with different Taper Constant $\mathrm{b} 2$ and constant Aspect Ratio $(\mathrm{a} / \mathrm{b}=1.5)$.

$\beta_{1}$. Here decrease in time period $\mathrm{K}$ as taper constant $\beta_{1}$ increases for both the modes of vibration is observed and the Fig. 3 clarifies the results of a steady decrease in time period K with increase in taper constants $\beta_{1}$.

In Fig. 4, the results of time period K, for first two modes of vibration for preset aspect ratio a/b $(=1.5)$, fixed thermal gradient $\alpha(=0.3)$ and for set value of taper constant $\beta_{1}(=0.4)$ but for different values of taper constant $\beta_{2}$. The decrease in time period $\mathrm{K}$ is indicated with increase in taper constant $\beta_{2}$ for both the modes of vibration.

Figures 5(a), 5(b) and 6(a), 6(b) respectively contain numerical values of deflection $w$ for preset aspect ratio a/b $(=1.5)$ for first two modes of vibration at initial time $0 . \mathrm{K}$ and at time $5 . \mathrm{K}$ for different values of $\mathrm{X}$ and $\mathrm{Y}$ for the subsequent cases:

(i) Fig. 5(a), 5(b): $\alpha=\beta_{1}=\beta_{2}=0.0, \alpha_{1}=0.2, \alpha_{2}=0.3$

(ii) Fig. 6(a), 6(b): $\alpha=0.2, \beta_{1}=0.3, \beta_{2}=0.4, \alpha_{1}=0.2, \alpha_{2}=0.3$ 

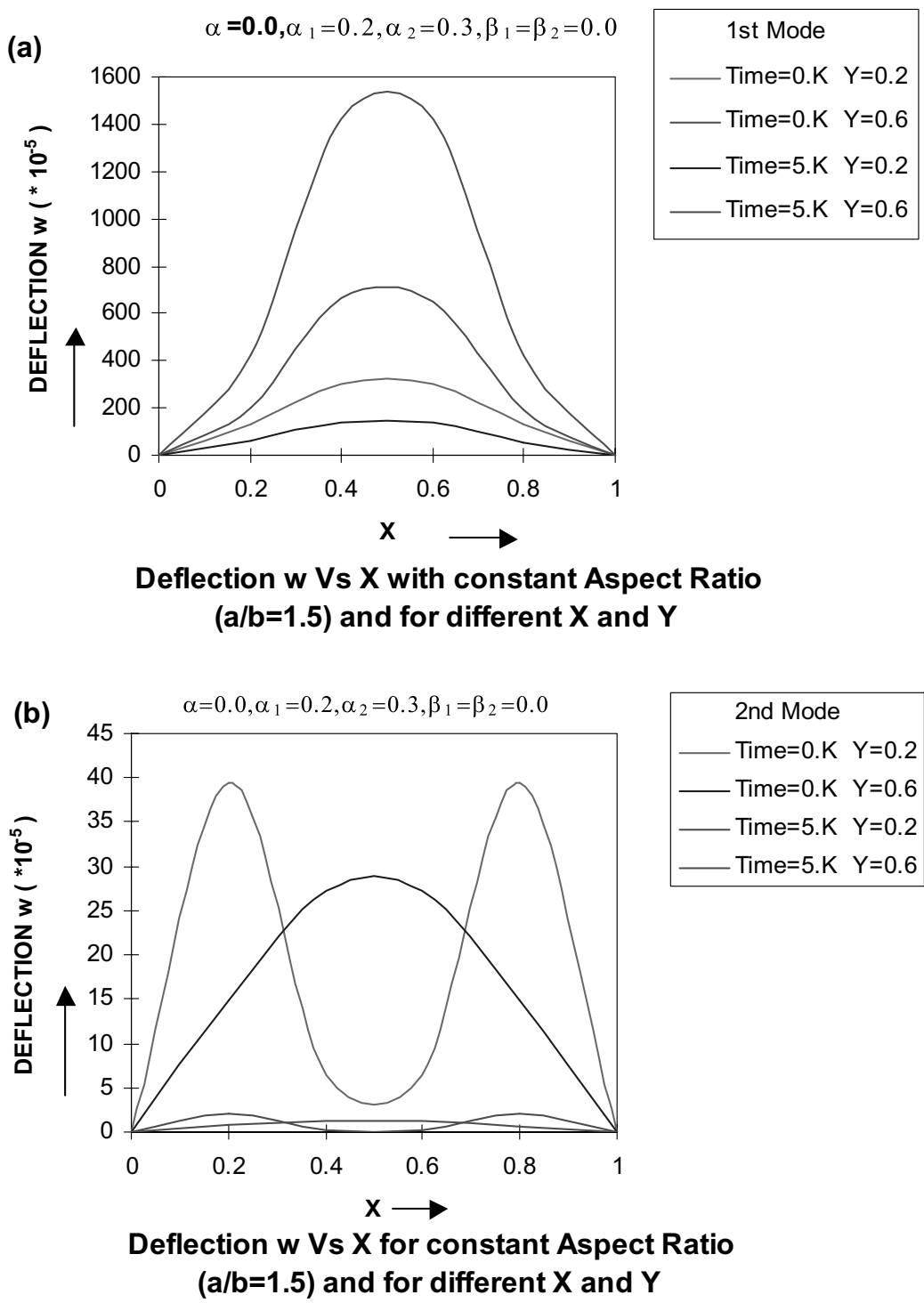

Fig. 5. (a) Deflection w Vs X with constant Aspect Ratio $(\mathrm{a} / \mathrm{b}=1.5)$ and for different $\mathrm{X}$ and $\mathrm{Y}$; (b) Deflection $\mathrm{w}$ Vs $\mathrm{X}$ for constant Aspect Ratio $(\mathrm{a} / \mathrm{b}=1.5)$ and for different $\mathrm{X}$ and $\mathrm{Y}$.

It is clearly observed from figs. that the deflection $\mathrm{w}$ for first mode of vibration first increases and then decreases till zero as $\mathrm{X}$ increases for different values of $\mathrm{Y}$ in every one of the above cases.

For the second mode of vibration, for $\mathrm{Y}=0.2$, an increase of deflection $\mathrm{w}$ followed by decrease followed by increase and finally zero is observed in all these figs. but for $\mathrm{Y}=0.6$, deflection $\mathrm{w}$ first increases and then decreases till zero as $\mathrm{X}$ increases.

Figure 7 shows the variation of deflection w for different values of aspect ratio $\mathrm{a} / \mathrm{b}$ and for preset values of $\mathrm{X}(=$ $0.2)$ and $\mathrm{Y}(=0.2)$ at initial time $0 . \mathrm{K}$ and at time $5 . \mathrm{K}$ for $\alpha=0.3, \alpha_{1}=0.2, \alpha_{2}=0.3, \beta_{1}=0.3, \beta_{2}=0.4$ for first two modes of vibration.

Figure 7 describes that deflection w continuously increases for first mode of vibration at initial time $0 . \mathrm{K}$ and first increases and then to some extent decreases at the time 5.K but an increase followed by decrease is observed for second mode of vibration, with increase in aspect ratio $\mathrm{a} / \mathrm{b}$, at initial time $0 . \mathrm{K}$ and at time $5 . \mathrm{K}$ both.

Here 0.K means at zero time periods and 5.K means at five times time period. 


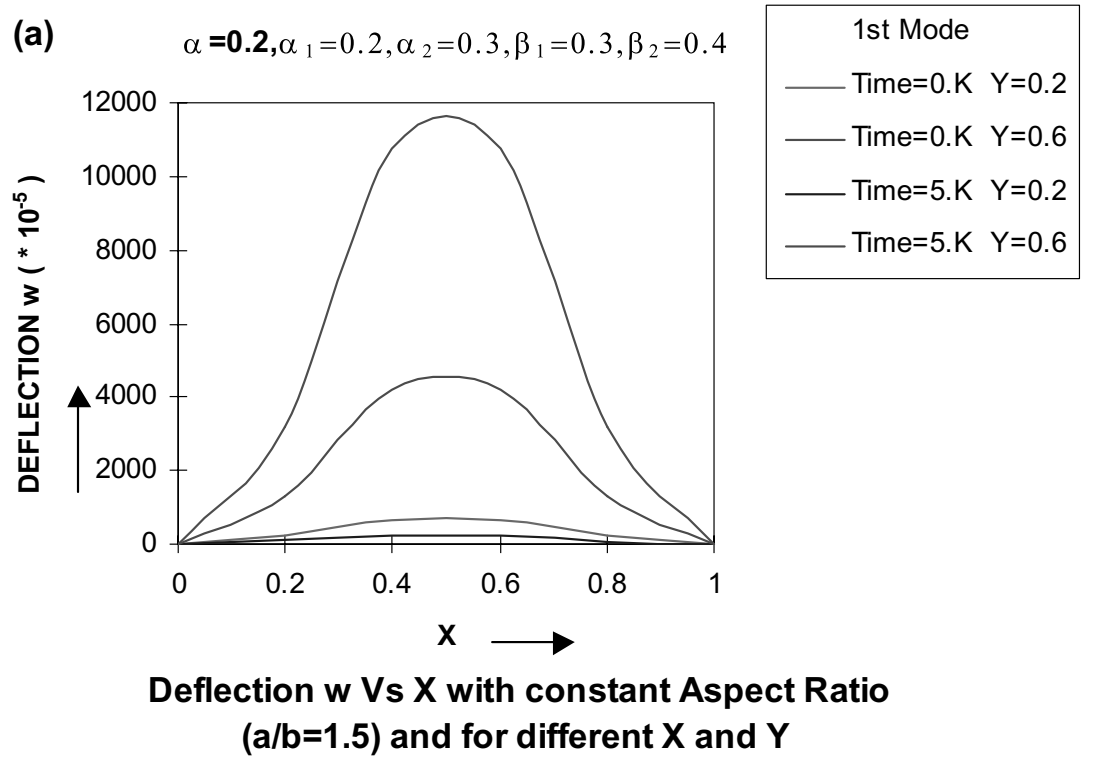

(b) $\quad \alpha=0.2, \alpha_{1}=\alpha_{2}=0.0, \beta_{1}=0.3, \beta_{2}=0.4$
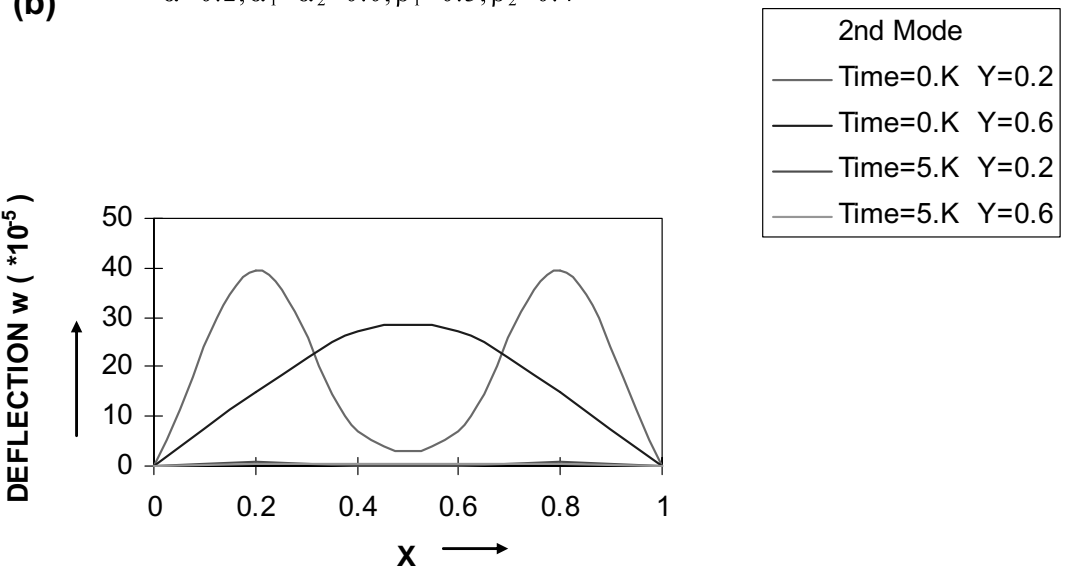

\section{Deflection w Vs X for constant Aspect Ratio} $(a / b=1.5)$ and for different $X$ and $Y$

Fig. 6. (a) Deflection w Vs X with constant Aspect Ratio $(\mathrm{a} / \mathrm{b}=1.5)$ and for different $\mathrm{X}$ and $\mathrm{Y}$; (b) Deflection w Vs X with constant Aspect Ratio $(\mathrm{a} / \mathrm{b}=1.5)$ and for different $\mathrm{X}$ and $\mathrm{Y}$.

\section{Conclusion}

The results for uniform unheated isotropic visco-elastic rectangular plate are compared to the published paper of the authors [8] and results for unheated visco-elastic rectangular plate are compared with the authors published paper [7] and both found to be in close agreement. Also the results for frequency are compare with [2,17] for aspect ratio $\mathrm{a} / \mathrm{b}=1.0$ and $\mathrm{b} / \mathrm{a}=0.5$ for Poisson ratio $\mathrm{v}=0.3$ and found in very good agreement. Further it is interesting to note that effect of taper in $\mathrm{x}$-direction is more dominate in comparison to $\mathrm{y}$-direction.

Therefore engineers can see and develop the plates in the manner so that they can fulfill the requirements.

Comparison result of time period for unheated plate with reference $[7,8]$ for $a / b=1.5$ for

(i) $\beta_{1}=0.0, \beta_{2}=0.0$

Ist Mode: 0.006679 (ours), 0.006679 ([8]), 0.006679 ([7]) 
$\alpha=0.3, \alpha_{1}=0.2, \alpha_{2}=0.3, \beta_{1}=0.3, \beta_{2}=0.4$

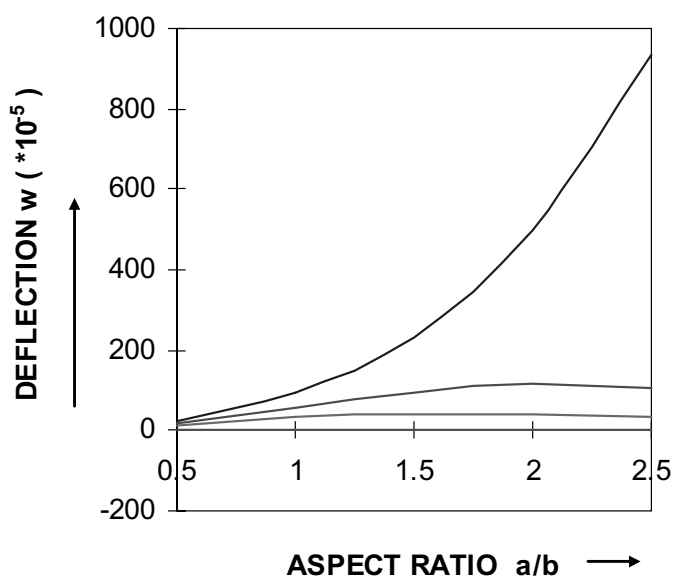

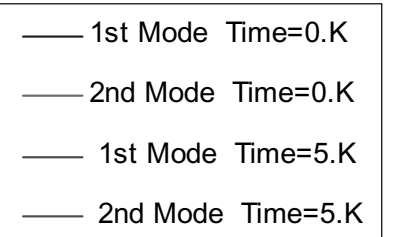

2nd Mode Time $=5 . \mathrm{K}$

\section{Variation of Deflection w for different Aspect}

Ratio $(\mathrm{a} / \mathrm{b})$ and for $\mathrm{X}=\mathrm{Y}=\mathbf{0 . 2}$

Fig. 7. Variation of Deflection $w$ for different Aspect Ratio $(a / b)$ and for $X=Y=0.2$.

IInd Mode: 0.001690 (ours), 0.001690 ([8]), 0.001690 ([7])

(ii) $\beta_{1}=0.0, \beta_{2}=0.2$

Ist Mode: 0.006163(ours), 0.006163 ([7])

Ind Mode: 0.001559 (ours), 0.001559 ([7])

(iii) $\beta_{1}=0.4, \beta_{2}=0.0$

Ist Mode: 0.005906 (ours), 0.005906 ([7])

IInd Mode: 0.001505 (ours), 0.001504 ([7])

(iv) $\beta_{1}=0.4, \beta_{2}=0.2$

Ist Mode: 0.005456(ours), 0.005456 ([7])

IInd Mode: 0.001389 (ours), 0.001389 ([7])

Comparison result of frequency for unheated uniform plate with reference $[2,17]$ for $a / b=1.0$ for Poisson ratio $\mathrm{v}=0.3$

Ist Mode: 36.002 (ours), 35.988 ([17]), 35.986 ([2])

IInd Mode: 73.545 (ours), 73.398 ([17]), 73.395 ([2])

Comparison result of frequency for unheated uniform plate with reference [17] for $b / a=0.5$ for Poisson ratio $\mathrm{v}=\mathbf{0 . 3}$

Ist Mode: 98.389(ours), 98.317 ([17])

IInd Mode: 127.351(ours), 127.310([17])

\section{References}

[1] D.V. Bambill and P.A.A. Laura, Fundamental frequency of transverse vibration of a clamped rectangular plate of cylindrical orthotropy, $J$ Sound and Vibration 220(3) (1999), 571-576. 
[2] R.B. Bhat, Natural frequencies of rectangular plates using characteristic orthogonal polynomials in Rayliegh-Ritz method, $J$ Sound and Vibration 102 (1985), 493-499.

[3] N.S. Bhatnagar and A.K. Gupta, Thermal effect on vibration of viscoelastic elliptic plate of variable thickness, Proc. of International Conference on Modelling and Simulation, Melbourne, 1987, 424-429.

[4] N.S. Bhatnagar and A.K. Gupta, Vibration analysis of viscoelastic circular plate subjected to thermal gradient, Modeling, Simulation and Control B15(1) (1988), 17-31, AMSE Press.

[5] H.L. Cox and J. Boxer, Vibration of rectangular plates point supported at the corners, Aeron Quart 11(1) (1960), 41-50.

[6] C. Filipich, P.A.A. Laura and R.D. Santos, A note on the vibrations of rectangular plates of variable thickness with two opposite simply supported edges and very general boundary conditions on the other two, J Sound and Vibration 50(3) (1977), 445-454.

[7] A.K. Gupta and A. Khanna, Vibration of clamped visco-elastic rectangular plate with parabolic thickness variations, J Shock and Vibration 15(6) (2008), 713-723.

[8] A.K. Gupta and A. Khanna, Vibration of viscoelastic rectangular plate with linearly thickness variations in both directions, $J$ Sound and Vibration 301(3-5) (2007), 450-457.

[9] R.K. Jain and S.R. Soni, Free vibrations of rectangular plates of parabolically varying thickness, Indian J Pure App Math A (1973), 267-277.

[10] H.A. Larrondo, D.R. Avalos, P.A.A. Laura and R.E. Rossi, Vibration of simply supported rectangular plates with varying thickness and same aspect ratio cutouts, J Sound and Vibration 244(4) (2001), 738-746.

[11] P.A.A. Laura, R.O. Grossi and G.I. Carneiro, Transverse vibrations of rectangular plates with thickness varying in two directions and with edges elastically restrained against rotation, $J$ Sound and Vibration 63(4) (1979), 499-505.

[12] A.W. Leissa, Vibration of plates, NASA SP-160, U.S. Govt. Printing office, 1969.

[13] K. Nagaya, Vibrations and dynamic response of viscoelastic plates on non-periodic elastic supports, J Engg for Industry 99 (1977), 404-409.

[14] W. Nowacki, Thermo Elasticity, Pergamon Press, New York, NY, 1962.

[15] R.E. Rossi, Transverse vibrations of thin, orthotropic rectangular plates with a rectangular cutouts with fixed boundaries, $J$ Sound and Vibration 221(4) (1999), 733-776.

[16] R.K. Sharma, Some Vibration Problems of Orthotropic Plates and Shells, PhD Thesis, University of Roorkee, 1977.

[17] B. Singh and S. Chakarverty, Flexural vibration of skew plates using boundary characteristic orthogonal polynomials in two variables, $J$ Sound and Vibration 173(2) (1994), 157-178.

[18] B. Singh and V. Saxena, Transverse vibration of rectangular plate with bi-directional thickness variation, J Sound and Vibration 198(1) (1996), 51-65.

[19] Z. Sobotka, Free vibration of visco-elastic orthotropic rectangular plates, Acta.Technica, CSAV, No.6, 1978, 678-705.

[20] R.L. Taylor and S. Govindjee, Solution of clamped rectangular plate problems, Technical Report, UCB/SEMM, $09,2002$.

[21] J.S. Tomar and A.K. Gupta, Effect of thermal gradient on frequencies of an orthotropic rectangular plate whose thickness varies in two directions, J Sound and Vibration 98(2) (1985), 257-262.

[22] J.S. Tomar and A.K. Gupta, Thermal effect on frequencies of an orthotropic rectangular plate of linearly varying thickness, $J$ Sound and Vibration 90(3) (1983), 325-331.

[23] D. Young, Vibration of rectangular plates by the Ritz method, J App Mech, Trans ASME 17(4) (1950), $448-453$. 

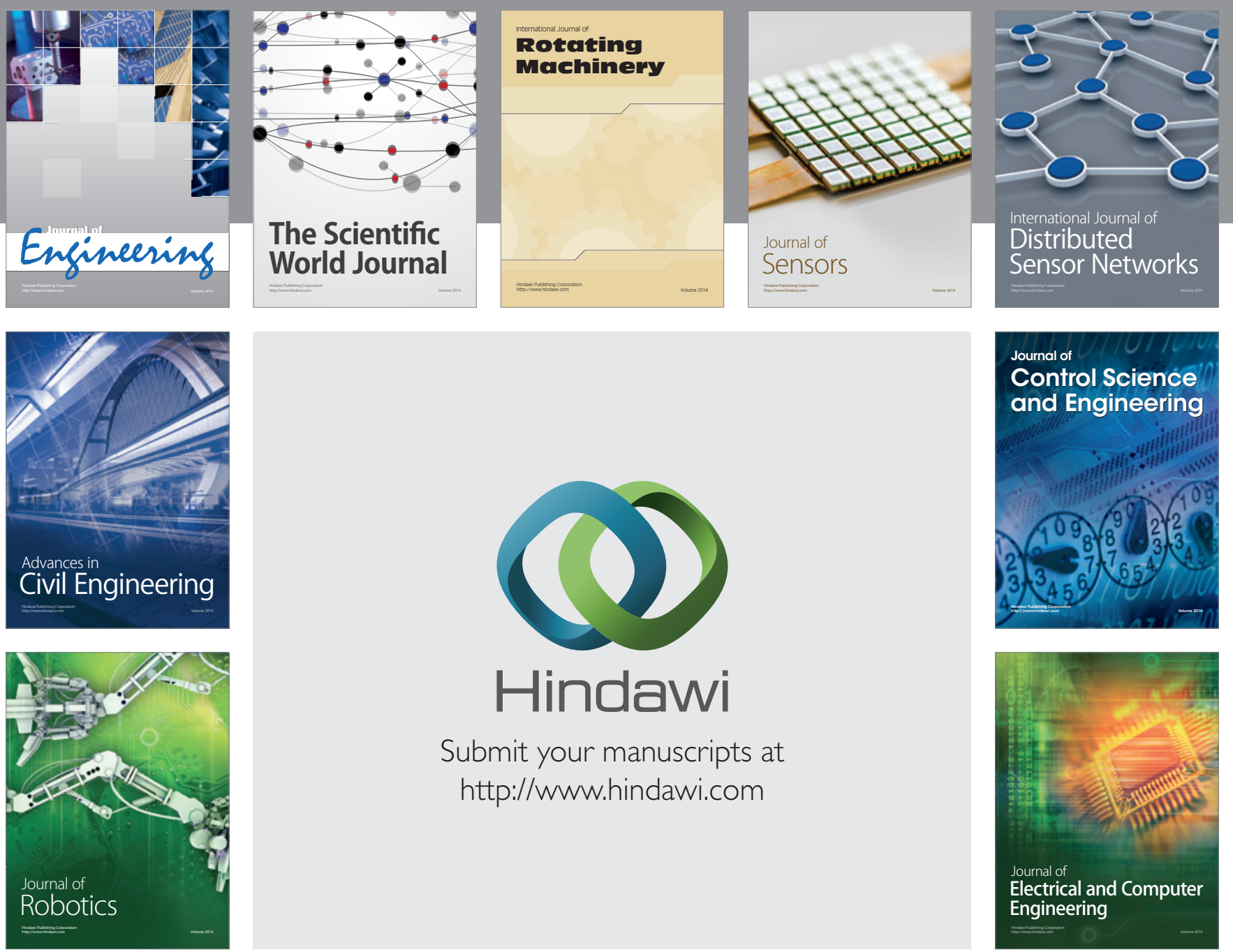

Submit your manuscripts at

http://www.hindawi.com
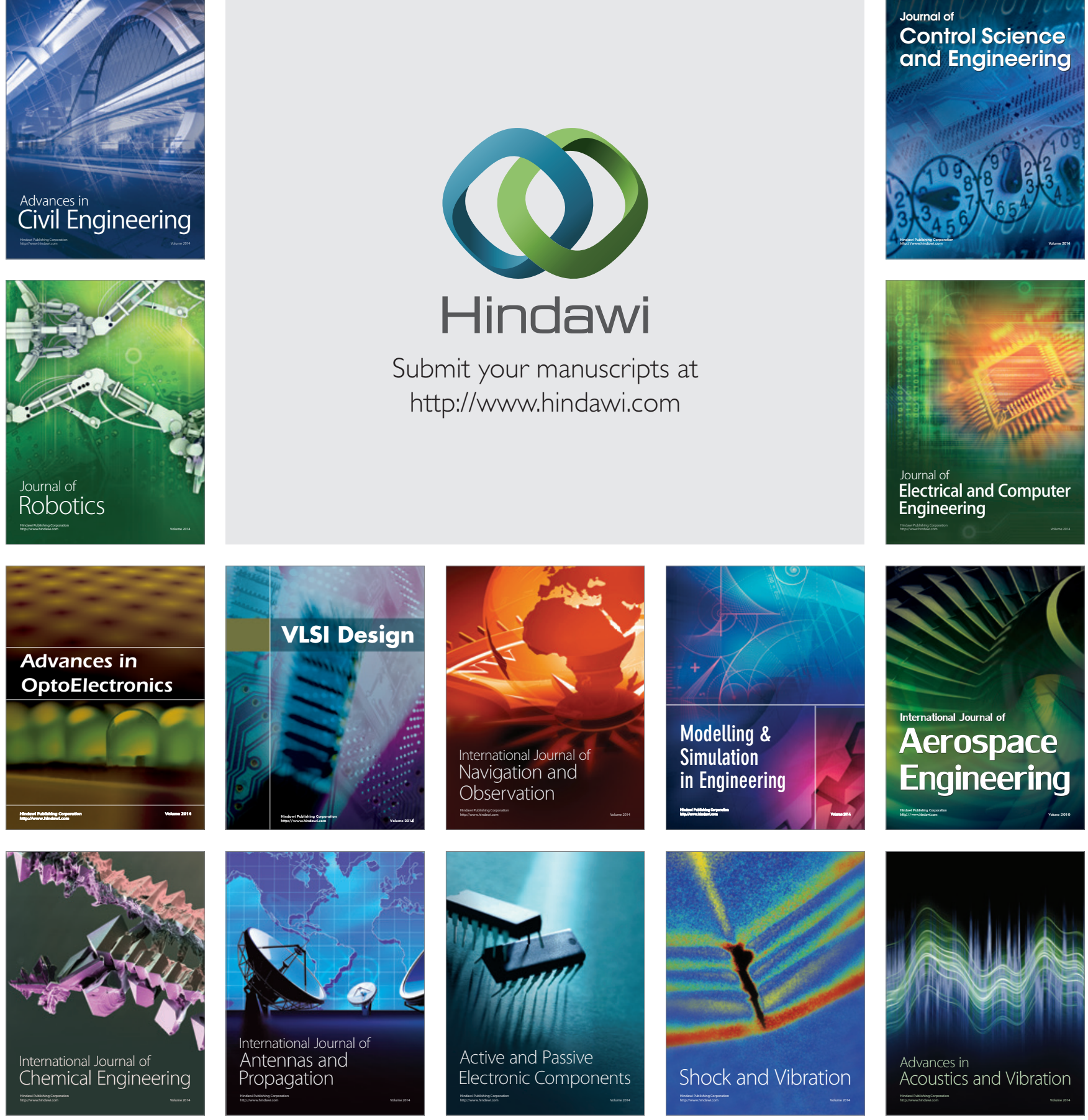